\title{
Neutrinos from gamma-ray bursts as a tool to explore quantum-gravity-induced Lorentz violation
}

\author{
URI JACOB* AND TSVI PIRAN* \\ Racah Institute for Physics, The Hebrew University, Jerusalem, 91904, Israel \\ *e-mail: uriyada@phys.huji.ac.il; tsvi@phys.huji.ac.il
}

Lorentz-invariance violation (LIV) arises in various quantumgravity $^{1,2}$ theories, but typically at Planck energies that are not accessible on Earth. To test LIV, we must turn to astronomical observations ${ }^{2-11}$. Time-of-flight measurements from astronomical sources have set the present limits on the LIV energy scale. According to existing models, gamma-ray bursts (GRBs) are accompanied by very high-energy neutrinos ${ }^{12,13}$. At these energies, the background level in neutrino detectors such as IceCube (currently under construction in Antarctica) is extremely low. We show that the detection of even a single neutrino from the same direction as a GRB, months after the burst, would be statistically significant and imply that the neutrino was associated with the burst. The detection of several delayed neutrinos from different bursts with compatible relations between their delay times, energies and distances would enable us to generically determine (or set limits on) LIV at levels that cannot be reached by any other method.

History tells us that symmetries, observed over a large range of parameters and believed to be fundamental properties of our physical world, may lose their significance later when observations are made over a larger range of parameters and when a new physical understanding arises. Such apparent symmetries often emerge as leading-order approximations of more complex symmetries, found to describe more accurately the larger range of observations. The Lorentz symmetry might be such an apparent symmetry, as Lorentz-invariance might be violated or deformed at very high energies. This possibility, which was initially motivated by attempts ${ }^{14-16}$ to resolve the GZK paradox ${ }^{17,18}$, arises naturally in various theories of quantum gravity (see, for example, refs 1,2 for a review).

Here, we consider a simple phenomenological approach ${ }^{16}$ for LIV with a symmetry-breaking energy scale of $\xi E_{\mathrm{pl}}$ ( $E_{\mathrm{pl}}$ being the Planck energy $\left.\sim 1.2 \times 10^{28} \mathrm{eV}\right)$. We consider a single LIV scale $(\xi)$ for all particles. This arises naturally in any theory in which the modification originates from a small-scale structure of spacetime. Certain theories, for example the one proposed by Coleman and Glashow $^{15}$, allow different LIV scales for different particles. In such a case, the time delay between low-energy photons and high-energy neutrinos, which we consider here, essentially limits the neutrinos LIV scale.
Taking into account only the leading-order correction, we expect, for particles with $E \ll \xi E_{\mathrm{pl}}$, a generic approximate dispersion relation:

$$
E^{2}-p^{2} c^{2}-m^{2} c^{4} \simeq \pm E^{2}\left(\frac{E}{\xi_{n} E_{\mathrm{pl}}}\right)^{n} .
$$

$E, p$ and $m$ denote the particle energy, momentum and mass, respectively. $c$ is the 'conventional' speed of light constant, which may be seen in our context as the speed of low-energy massless particles. Assuming that the standard relation $v=\mathrm{d} E / \mathrm{d} p$ holds, the $+(-)$ sign accounts for superluminal (infraluminal) motion. Note, the increase in the reactions' thresholds that resolves the GZK paradox requires the - sign.

The most generic attempts to constrain the LIV scale are based on the energy-dependent delay in the arrival of high-energy particles $^{2}$ (loosely speaking, we use the term delay to imply both a delay and an early arrival, corresponding to the - and + signs in equation (1)). As no delays have been observed in $\mathrm{GRBs}^{3-6}$, flaring active galactic nuclei ${ }^{7}$ (AGNs), or $\mathrm{GeV}$ emission from the Crab pulsar ${ }^{8}$, we have only lower bounds: $\xi_{1} \gtrsim 0.01$ from GRBs and $\xi_{2} \gtrsim 10^{-9}$ from flaring AGNs (this limit for $\xi_{2}$ is obtained by translating the limits given in ref. 7 for $n=1$ to $n=2$ ). Limits for higher values of $n$ are too small to be of any relevance. Stronger LIV bounds (see, for example, refs 9-11) can be obtained within specific theories for LIV. However, such limits are valid only for the specific theory assumed. Time-of-arrival analyses, which we discuss here, provide the most generic model-independent LIV test ${ }^{19}$.

The time delay of a particle with energy $E$, arriving from a source at a distance $d$, is of the order: $\Delta t \approx(d / c)\left(E / \xi_{n} E_{\mathrm{pl}}\right)^{n}$ (see equation (2) for an exact formula). To improve the current limits, we need a more distant source, an observation at higher energies or an improved temporal resolution (provided that the particles are emitted simultaneously at the source). However, pair production on the infrared background limits the distances that high-energy photons can travel. In addition, the lower photon number fluxes at higher energies limit the possible time resolution ${ }^{20}$. Very high-energy neutrinos ${ }^{21}$ provide an alternative that overcomes these problems. 


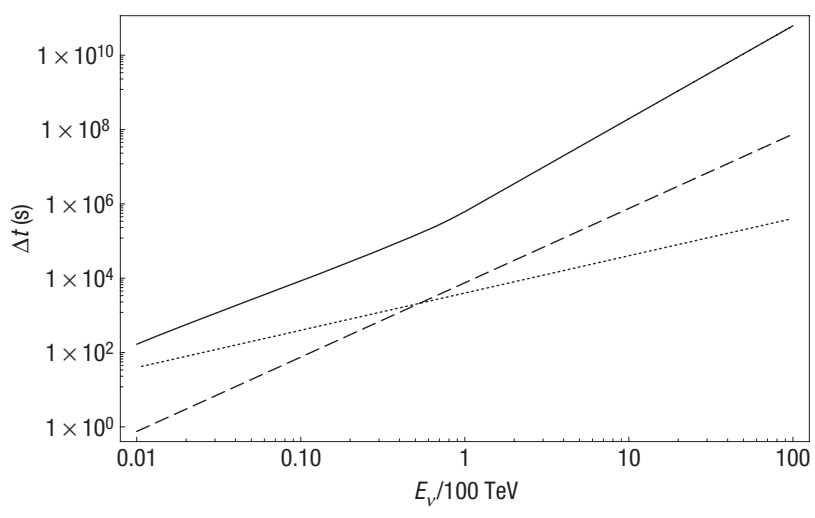

Figure 1 LIV delay periods versus 'background-free' intervals. As a function of neutrino energy: $t_{b}(E, 0.01)$ (solid line), the time interval for $10^{-4}$ background events (corresponding to a false positive of $1 \%$ ) and the LIV time delays, $\Delta t$, from $z=1$ with $\xi_{1}=1$ (dotted line) or $\xi_{2}=10^{-7}$ (dashed line).

Practically all current GRB models (see ref. 22 for a review) predict that bursts of very high-energy neutrinos, with energies ranging from $100 \mathrm{TeV}$ to $10^{4} \mathrm{TeV}$ (and possibly up to $10^{6} \mathrm{TeV}$ ) should accompany GRBs ${ }^{12,13}$. As the energies of these neutrinos are orders of magnitude higher than the energies of photons observed from cosmological distances, the corresponding time delays are orders of magnitude longer and can open a new window on the LIV parameter space.

High-energy-neutrino detectors of $\mathrm{km}^{3}$ scale, which are required for the observation of GRB neutrinos, are currently under construction. The IceCube observatory, which will expand the AMANDA detector, operational in the Antarctic ice, to an effective volume larger than a cubic kilometre, is already taking data and is scheduled for completion in 2010. It uses a large volume of natural ice as a Cherenkov detector. High-energy neutrinos interact with the ice and produce secondary particles whose Cherenkov radiation enables us to measure the neutrinos' energy and direction. Similar detectors (ANTARES, NESTOR) that use water instead of ice are under construction in the Mediterranean. Additional projects, intended to explore other neutrino signatures such as air showers, have also been initiated, and will detect even higher energy neutrinos ${ }^{23}$.

The 1,637 bursts detected over an effective exposure time of 2.62 years and recorded in the BATSE 4B Catalog have an average fluence of $1.2 \times 10^{-5} \mathrm{ergs} \mathrm{cm}^{-2}$. Assuming that the emitted neutrinos fluence is one tenth of that observed in photons (a reasonable assumption concerning the relevant interactions), we obtain an average GRB-induced neutrino, $v$, flux of $5 \times 10^{18} \mathrm{eV} \mathrm{km}^{-2} \mathrm{yr}^{-1}$. Using the most likely value of $E_{v} \approx 100 \mathrm{TeV}$ and a detection probability of $10^{-4}$ in a $\mathrm{km}^{3}$ detector, we estimate a detection rate of five events per year. This rough estimate is in agreement with several detailed model-dependent calculations ${ }^{24-26}$ that find a detection rate of a few to a few dozen events per year. For a given total emitted neutrino energy, the number of emitted neutrinos decreases proportionally to the average energy. However, the detectors' sensitivity increases for higher energy neutrinos ${ }^{23,25}$, and this compensates somewhat over the decreasing particle flux, so the detected flux decreases only as $E_{v}^{-0.5}$ for $E_{v} \gtrsim 100 \mathrm{TeV}$. Thus, for IceCube, only neutrinos up to approximately $10^{4} \mathrm{TeV}$ are relevant.

Choubey and $\mathrm{King}^{21}$ proposed to compare the arrival times of GRB-associated neutrinos to test Lorentz violation. However, the detection probability of a single neutrino from a given burst is small, $\sim 10^{-2}$ (the detection probability may reach 0.1 in an

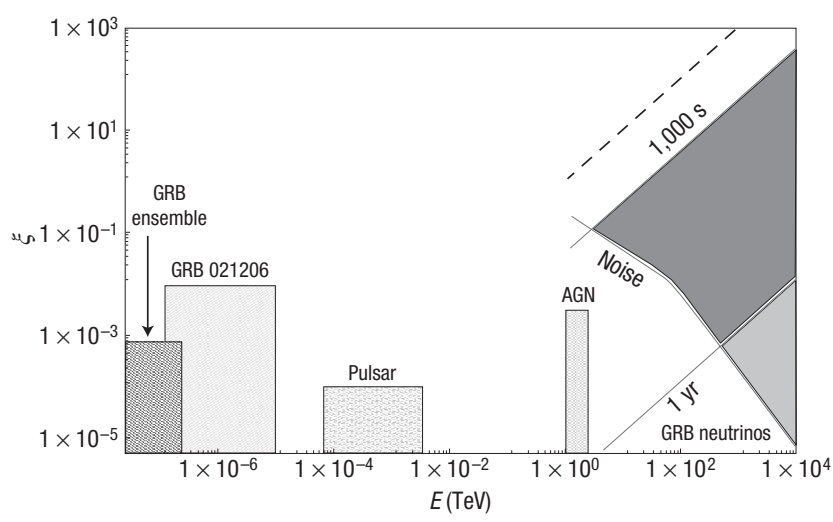

Figure 2 The examinable $\xi_{1}$ parameter space. The range of $\xi_{1}$ that can be explored for LIV delays using GRB neutrinos from $z=1$ as a function of $E_{v}$. The grey regions indicate the range where $1,000 \mathrm{~s}<\Delta t<t_{b}(E, 0.01)$. The additional condition $\Delta t<1 \mathrm{yr}$ is imposed in the upper right region. The dashed line describes the lower limit that can be obtained from a simultaneous (within $30 \mathrm{~s}$ ) detection of a high-energy neutrino and the prompt GRB. Also marked are current limits obtained using various photon sources.

extremely bright burst), and it is improbable that two neutrinos will be detected from the same burst. A direct comparison between the arrival time of two neutrinos ${ }^{21}$ from the same source is, therefore, unlikely. Certain GRB models predict that the neutrino luminosity is a nonlinear function of the burst's gamma-ray luminosity ${ }^{27}$. In this case, more than one neutrino might be detected from a specific burst. This would increase the confidence of association of the neutrinos with the GRB and will also enable a direct comparison. Here, we show that even without multiple detections from the same burst (that will happen only if a specific neutrino production model is correct), a comparison of the neutrino arrival time with the prompt low-energy GRB photons could be used to test $\operatorname{LIV}^{12,28}$. Choubey and King ${ }^{21}$ were concerned that such a comparison would be impractical because of the unknown delay between photon and neutrino emission. We show that the expected neutrino LIV delays are typically long enough and the background level in the neutrino detector is low enough to overcome this problem. Furthermore, statistical methods developed by Ellis et al. ${ }^{3,6,19}$ to combine data from different bursts and deal with intrinsic time delays in GRBs can be used here to overcome this uncertainty.

The LIV time delay of a massless particle with an observed energy $E$, emitted at redshift $z$ is:

$$
\Delta t=\frac{1}{H_{0}} \int_{0}^{z}\left(\frac{1+n}{2}\left(\frac{E}{\xi E_{\mathrm{pl}}}\right)^{n}\left(1+z^{\prime}\right)^{n}\right) \frac{\mathrm{d} z^{\prime}}{\sqrt{\Omega_{m}\left(1+z^{\prime}\right)^{3}+\Omega_{\Lambda}}} .
$$

$H_{0}, \Omega_{m}$ and $\Omega_{\Lambda}$ are the cosmological parameters evaluated today, to which we assign throughout our calculations the standard values. Delays of the order of an hour are expected for a $100 \mathrm{TeV}$ neutrino from a cosmological source at $z=1$ with $\xi_{1}=1$ (or with $\xi_{2}=10^{-7}$ ). These delays should be compared, first, with delays produced by the neutrino's rest mass, $m_{v}: \approx 10^{-11} \mathrm{~s}\left(E_{v} / 100 \mathrm{TeV}\right)^{-2}\left(m_{v} / 1 \mathrm{eV}\right)^{2}$ for a source at a cosmological distance $(z \approx 1)$. For $n=1$ and $\xi_{1}=1$ at $100 \mathrm{TeV}$, this delay (with $m_{v}=1 \mathrm{eV}$ ) is 14 orders of magnitude smaller than the LIV delay. We would need a neutrino mass of $10 \mathrm{MeV}$ to make the time delay due to the neutrino mass comparable to the LIV time delay. Even for $n=2$ and $\xi_{2}=1$, the LIV 
time delay is 10 times larger than the delay arising from a neutrino mass of $1 \mathrm{eV}$. As we are interested in neutrinos with higher energies and as the neutrino mass is expected to be even lower than $1 \mathrm{eV}$, we safely regard neutrinos as massless for our purposes.

To estimate the significance of the association of a delayed neutrino (of energy $E$ ) with the GRB, we compare the LIV delay values, $\Delta t$, with the time interval, $t_{\mathrm{b}}(E, p)$, in which (at a given confidence level, $p$ ) no background neutrino from the same direction in the sky is expected. The dominant background at most of the energy range arises from muons produced by atmospheric neutrinos. The decay of atmospheric charmed particles ${ }^{23}$ also contributes to the background. Unfortunately, the estimates of this contribution are uncertain ${ }^{29}$. Under the most severe assumptions ${ }^{29}$, this background dominates above $\sim 500 \mathrm{TeV}$. However, at these energies the background is so small that even this severe estimate is sufficiently low that qualitatively our results remain valid. Using the atmospheric neutrino spectrum ${ }^{25}\left(\propto E_{\nu}^{-\beta}\right)$ and the probability that a muon neutrino generates a detectable muon ${ }^{25}\left(\propto E_{v}^{\alpha}\right)$, we estimate the number of background events above an energy $E$ detected in a detector of size $A$ (in $\mathrm{cm}^{2}$ ), from a solid angle $\Omega$ and during a time interval $t$ (in seconds) as:

$$
N_{\mathrm{bg}} \simeq 5 \times 10^{-17} A \cdot \Omega \cdot t \int_{E}^{\infty} \mathrm{d} \tilde{E}_{\nu} \tilde{E}_{v}{ }^{\alpha-\beta}
$$

where $\tilde{E}_{v} \equiv E_{v} / 100 \mathrm{TeV},(\alpha=1, \beta=3.7)$ for $E_{v}<100 \mathrm{TeV}$ and $(\alpha=0.5, \beta=4)$ for $E_{v}>100 \mathrm{TeV}$. IceCube is designed to determine the direction of muons with sub-degree accuracy $^{23}$, thus we take $\Omega \approx 10^{-3}$ square radians. Figure 1 shows $t_{\mathrm{b}}(E, 0.01)$, the interval in which we expect $10^{-4}$ background events (corresponding to a rate of false alarm of 1\%). An observed neutrino can be associated with a burst (and interpreted as a positive detection of a time delay) if $t_{\mathrm{b}}(E, p)>\Delta t$. As the detector is extremely quiet at these energies, a neutrino arriving from the same direction in the sky months or even years after gamma rays can be associated with the burst. However, for practical purposes we set an arbitrary bound on the maximal delay as a year. For $\xi_{1} \gtrsim 1\left(\xi_{2} \gtrsim 10^{-7}\right)$, the background does not pose any problem. Thus, for $n=1$ we can explore, using GRB neutrinos, the parameters up to and above the Planck scale, a region that cannot be explored in any other way today ${ }^{20}$.

The uncertainty concerning the simultaneity of the prompt gamma-rays and the expected neutrinos' burst, limits the detectability of LIV time delays between the photons and the neutrinos. Depending on the properties of the burst and on the neutrino production model, there might be an intrinsic delay between the prompt GRB and the time of neutrino emission. In practically all current GRB models, the expected neutrinos are produced in high-energy collisions that take place while the Lorentz factor of the outgoing ejecta is large. These conditions are satisfied during the burst and during the $1,000 \mathrm{~s}$ after it. Thus, the intrinsic delay is generally expected to be of this order of magnitude or lower.

To appreciate the influence of this uncertainty, for illustrative purposes, we impose a conservative minimal delay of $1,000 \mathrm{~s}$. Clearly, because of this intrinsic delay and of other potential problems, no matter what the statistical significance of the detection of a single delayed neutrino would be, it will not be considered on its own as serious evidence for LIV. However, several such detections are expected per year. The detection of several delayed neutrinos from different bursts would provide an ensemble of arrival times, energies and distances. If the delays arise from LIV, the relations between these quantities predicted in equation (2) should be apparent in the data. In particular, the delay should depend linearly on the distance (with the appropriate redshift corrections), whereas we do not expect any correlation between

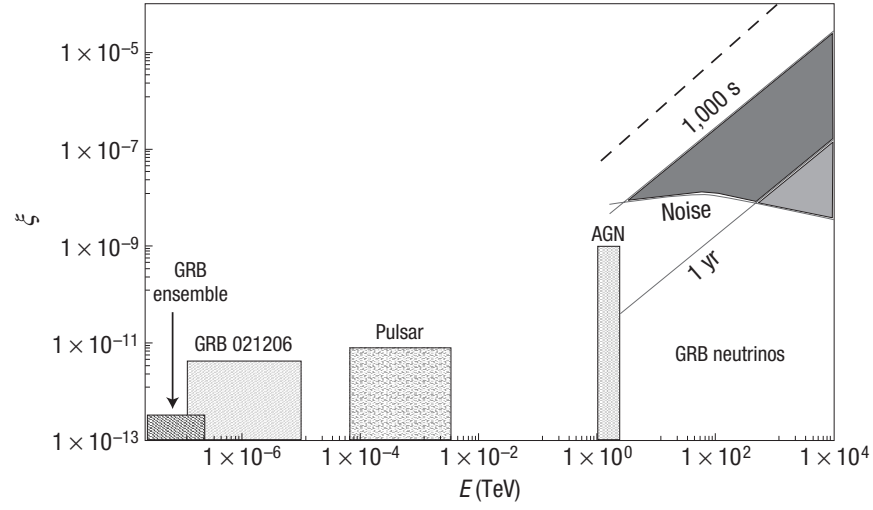

Figure 3 The examinable $\xi_{2}$ parameter space. The range of $\xi_{2}$ that can be explored for LIV delays using GRB neutrinos from $z=1$ as a function of $E_{v}$. The grey regions indicate the range where $1,000 \mathrm{~s}<\Delta t<t_{b}(E, 0.01)$. The additional condition $\Delta t<1 \mathrm{yr}$ is imposed in the upper right region. The dashed line describes the lower limit that can be obtained from a simultaneous (within $30 \mathrm{~s}$ ) detection of a high-energy neutrino and the prompt GRB. Also marked are current limits obtained using various photon sources.

the delay and the distance if the delay is intrinsic. The energy dependence should be determined by the factor $n$, which should be the same, of course, for all cases. Following the methods developed by Ellis et al. ${ }^{3,6,19}$ for analysing GRB data (in which intrinsic delays between high- and low-energy photons pose a serious problem), a combined analysis of this ensemble could provide a solid proof of the dependence of the delay on the distance and the energy as given by equation (2) and this can confirm or rule out LIV.

Figures 2 and 3 show the ranges of $\xi_{1,2}$ that can be determined by a delayed detection of GRB neutrinos. These ranges are many orders of magnitude above the current limits. The detection of neutrinos coinciding with the prompt GRB observation will set new upper limits to the LIV scale (see Figs 2 and 3). No detection at all will, of course, send us back to revise current GRB models.

Given the sky coverage of the GRB detectors and of IceCube, we expect a few associations between a GRB and a neutrino event per year. Repeated observations over several years of neutrinos associated with GRBs with compatible arrival times, distances and energies might be our best way to detect or rule out LIV over the most interesting regions of the parameters phase space.

Received 13 July 2006; accepted 30 November 2006; published 21 January 2007.

\section{References}

1. Amelino-Camelia, G. Quantum theory's last challenge. Nature 408, 661-664 (2000).

2. Amelino-Camelia, G. et al. Tests of quantum gravity from observations of gamma-ray bursts. Nature 393, 763-765 (1998).

3. Ellis, J., Mavromatos, N. E., Nanopoulos, D. V. \& Sakharov, A. S. Quantum-gravity analysis of gamma-ray bursts using wavelets. Astron. Astrophys. 402, 409-424 (2003).

4. Boggs, S. E., Wunderer, C. B., Hurley, K. \& Coburn, W. Testing Lorentz invariance with GRB 021206 Astrophys. J. Lett. 611, L77-L80 (2004).

5. Rodríguez Martínez, M., Piran, T. \& Oren, Y. GRB 051221A and tests of Lorentz symmetry. J. Cosmol. Astropart. Phys. 05(2006)017 (2006).

6. Ellis, J. et al. Robust limits on Lorentz violation from gamma-ray bursts. Astropart. Phys. $\mathbf{2 5}$ 402-411 (2006).

7. Biller, S. D. et al. Limits to quantum gravity effects on energy dependence of the speed of light from observations of TeV flares in active galaxies. Phys. Rev. Lett. 83, 2108-2111 (1999).

8. Kaaret, P. Pulsar radiation and quantum gravity. Astron. Astrophys. 345, L32-L34 (1999).

9. Jacobson, T., Liberati, S. \& Mattingly, D. A strong astrophysical constraint on the violation of special relativity by quantum gravity. Nature 424, 1019-1021 (2003).

10. Jacobson, T., Liberati, S., Mattingly, D. \& Stecker, F. W. New limits on Planck scale Lorentz violation in QED. Phys. Rev. Lett. 93, 021101 (2004).

11. Stecker, F. W. \& Scully, S. T. Lorentz invariance violation and the spectrum and source power of ultrahigh energy cosmic rays. Astropart. Phys. 23, 203-209 (2005). 
12. Waxman, E. \& Bahcall, J. High energy neutrinos from cosmological gamma-ray burst fireballs. Phys. Rev. Lett. 78, 2292-2295 (1997).

13. Vietri, M. On the energy of neutrinos from gamma-ray bursts. Astrophys. J. 507, 40-45 (1998).

14. Gonzalez-Mestres, L. Physics opportunities above the Greisen-Zatsepin-Kuzmin cutoff: Lorentz symmetry violation at the Planck scale. AIP Conf. Proc. 433, 148-158 (1998).

15. Coleman, S. \& Glashow, S. L. High-energy tests of Lorentz invariance. Phys. Rev. D 59, 116008 (1999).

16. Amelino-Camelia, G. \& Piran, T. Planck-scale deformation of Lorentz symmetry as a solution to the ultrahigh energy cosmic ray and the TeV-photon paradoxes. Phys. Rev. D 64, 036005 (2001).

17. Greisen, K. End to the cosmic-ray spectrum? Phys. Rev. Lett. 16, 748-750 (1966).

18. Zatsepin, G. T. \& Kuzmin, V. A. Upper limit of the spectrum of cosmic rays. JETP Lett. 4 , 78-80 (1966).

19. Ellis, J., Mavromatos, N. E., Nanopoulos, D. V. \& Sakharov, A. S. Cosmology: Synchrotron radiation and quantum gravity. Nature $\mathbf{4 2 8},(2004)$.

20. Rodríguez Martínez, M. \& Piran, T. Constraining Lorentz violations with gamma ray bursts. J. Cosmol. Astropart. Phys. 04(2006)006 (2006).

21. Choubey, S. \& King, S. F. Gamma ray bursts as probes of neutrino mass, quantum gravity, and dark energy. Phys. Rev. D 67, 073005 (2003).

22. Piran, T. The physics of gamma-ray bursts. Rev. Mod. Phys. 76, 1143-1210 (2004).

23. Halzen, F. Astroparticle physics with high energy neutrinos: from AMANDA to IceCube. Eur. Phys. J. C 46, 669-687 (2006).

24. Waxman, E. Extra galactic sources of high energy neutrinos. Phys. Scripta T 121, 147-152 (2005).
25. Guetta, D. et al. Neutrinos from individual gamma-ray bursts in the BATSE catalog. Astropart. Phys. $20,429-455$ (2004).

26. Alvarez-Muñiz, J., Halzen, F. \& Hooper, D. W. High energy neutrinos from gamma ray bursts: Event rates in neutrino telescopes. Phys. Rev. D 62, 093015 (2000).

27. Halzen, F. \& Hooper, D. W. Neutrino event rates from gamma-ray bursts. Astrophys. J. Lett. 527, L93-L96 (1999).

28. Amelino-Camelia, G. Proposal of a second generation of quantum-gravity-motivated Lorentz-Symmetry tests: Sensitivity to effects suppressed quadratically by the Planck scale. Int. J Mod. Phys. D 12, 1633-1639 (2003).

29. Ahrens, J. et al. Sensitivity of the IceCube detector to astrophysical sources of high energy muon neutrinos. Astropart. Phys. 20, 507-532 (2004).

\section{Acknowledgements}

This research was supported by the ISF center of excellence for High Energy Astrophysics. Correspondence and requests for materials should be addressed to U.J.

Competing financial interests

The authors declare that they have no competing financial interests.

Reprints and permission information is available online at http://npg.nature.com/reprintsandpermissions/ 